\title{
Thermodynamic Characteristics of Solid
}

\author{
Zdzisław Pluta, Tadeusz Hryniewicz* \\ Koszalin University of Technology, Raclawicka 15-17, PL 75-620 Koszalin, Poland \\ *E-mail addres: Tadeusz.Hryniewicz@tu.koszalin.pl
}

„A proper word to the matter should be given!"

Cyprian Kamil Norwid

\begin{abstract}
The paper is to present the developed thermodynamic characteristics of a solid. The existent description of dependence of temperature on the proper entropy (thermal capacitance) was used as the beginning point of the considerations. A critical evaluation of the picture of that dependence was a consecutive link of the considerations. Furthermore, an adequate description of the discussed heat phenomenon, taking into account the detailed characteristics of the entropy growth characteristics, has been presented. This characteristics indicates a clear quantum character of the studied reality, reflected by the potentials, proper and gained, respectively. These magnitudes are the measures of the proper and gained specific energies of this thermodynamic factor. The dependence of temperature on the specific energy has been described to formulate a formula on the heat work. At the end the dependence between the specific heat and the entropy constant is derived. In conclusion, a quantum nature of macroscopic reality has been noticed together with the definition of the heat energy notion. The necessity of noticing the description of any other reality in this scale has been indicated.
\end{abstract}

Keywords: Thermodynamics; Solid; Thermodynamic characteristics; Interstate space; Potential field; Specific proper heat energy; Specific gained heat energy; Energetic band; Heat work; Source characteristics of entropy growth; Entropy constant

\section{INTRODUCTION}

One could presume that on the ground of thermodynamics, being - as mentioned in the literature [1] - the Amazon of notions, the energy notion has been defined and functions without an obstacle. Mirabile dictu (wonderful to relate), as it appears this notion has been featured with thermodynamic belongings, though having rather a formal meaning to the thermodynamics. That branch of science prescinds from the notion contents of energy. That is a strange approach to the energy notion and difficult to understand.

Sometimes even a definition of energy is neglected as non existent. The question arises then why this notion of energy, without a content, is in use. Is it an empty notion, or may be too difficult to define?

The Author of cited work [1] explains quite frankly as follows: „(...) It seems to be easier to understand what is the entropy than what is the energy. It is difficult to give a strict definition of energy. One may substantiate that it is the body ability to perform work or some 
property of the curved space-time, or even the curving itself. But frankly speaking, none of these definitions is sufficiently convincing; whereas the entropy understanding does not provide any difficulties (...)".

The notion of energy should be understood. One cannot tolerate such a cognitive discontinuity. It cannot be treated as a fundamental notion of the reality without its understanding.

That cognitive situation induced the Authors of this work to explain the energy notion. The heat energy, as the one having strict connection with thermodynamics, is to be discussed in the paper. It will be referred to solids, as the bodies being in the solid state of aggregation. The energetic states of solids will be joined with the state of aggregation.

\section{EXISTENT DESCRIPTION OF THE DEPENDENCE OF TEMPERATURE ON ENTROPY}

The starting point of considerations will be the existent dependence of temperature on entropy. Literature [2] provides a primary dependence of temperature $T$ on the specific entropy $s$, the dependence in the form of differential equation; then the solution of this dependence is presented, providing the graphic form; afterwards the graphic interpretation of the specific heat has been presented.

The mentioned differential equation (related to the assumption that the specific volume $v=i d e m$, and $d v=0$ ) has the following form:

$$
d s=\frac{c}{T} d T
$$

where: $d s$ - total differential of specific entropy, $d T$ - total differential of temperature, $c-$ specific heat, and $T$ - temperature.

The specific heat of a solid is assumed as constant, then $c=c_{0}$. Thus after integrating the equation (1), of the form as follows

$$
\frac{d T}{T}=\frac{1}{c_{0}} d s
$$

one obtains

$$
s=c_{0} \ln T+s_{0}
$$

That solution of the equation (1) is given in the literature [2]. Furthermore, this literature explains that the integration constant $s_{0}$ is determined, assuming the entropy of this thermodynamic factor at $T=273.15 \mathrm{~K}$ is equal zero (that time $\mathrm{K}$ is substituted for the Kelvin degree from the past). It results from equation (3) that $s_{0}=-c_{0} \ln 273.15$. After substituting this value to equation (3) one obtains 


$$
s=c_{0} \ln \frac{T}{273.15}
$$

The image of this equation is an exponential curve characteristic with the sub-tangent in any point to possess a constant value. More steep exponential line (Fig. 1) is referred to the body with a lesser specific heat. (The original form of this Fig. 1 has been detained.)

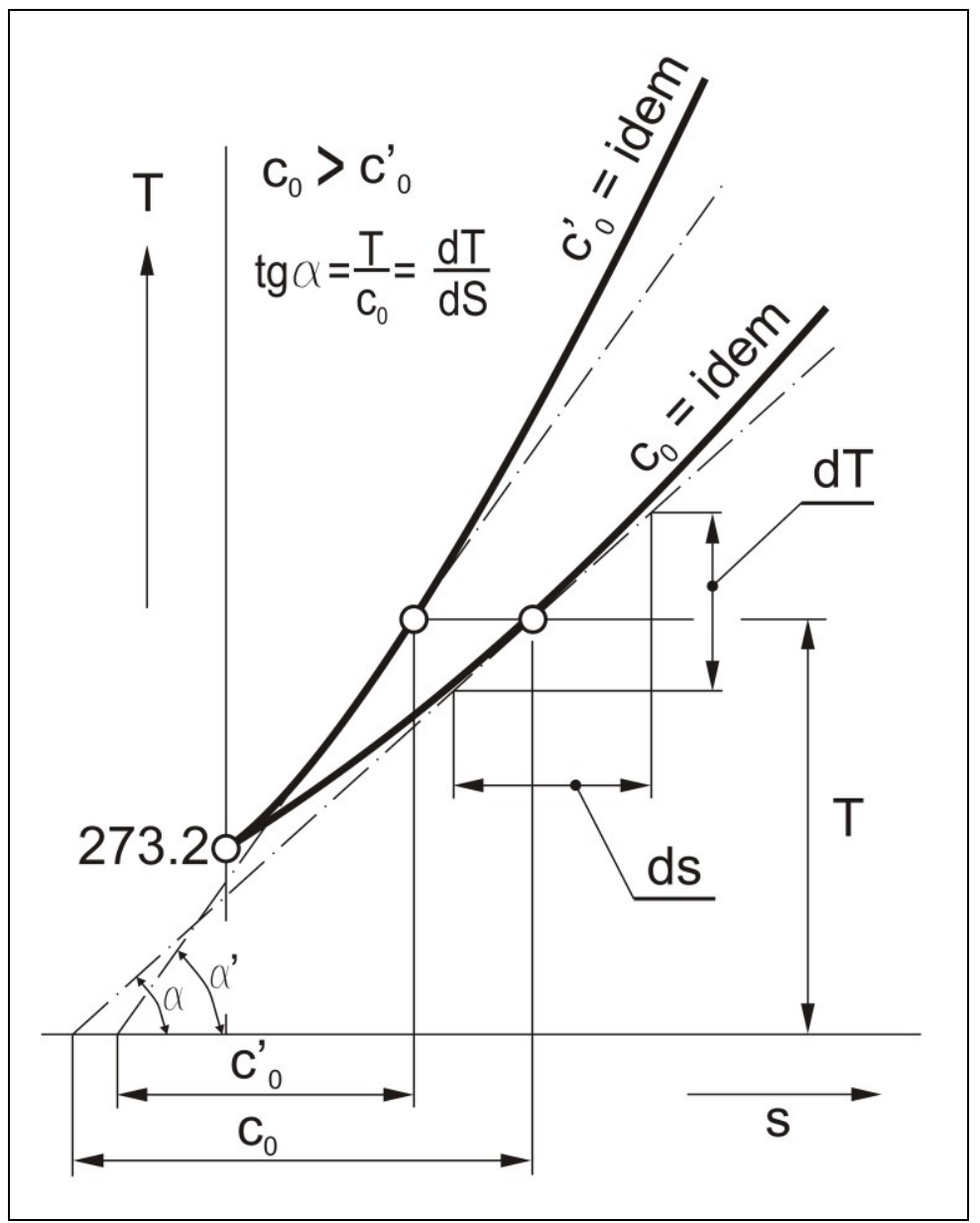

Fig. 1. $T, s$ plot for solid [2]

It is worth noticing that the coordinate system should correspond with the description of dependence $T=f(s)$, that is the equation (2). Let us consider the course of solution of this equation. After integrating of the both sides one obtains the result

$$
\ln T=\frac{1}{c_{0}} S+C^{*}
$$

or 


$$
T=e^{\frac{s}{c_{0}}+C^{*}}=e^{C^{*}} \cdot e^{\frac{s}{c_{0}}}=C e^{\frac{s}{c_{0}}}
$$

After regarding that for $s=0$ the temperature $T=T_{0}$, one obtains

$$
C=T_{0}
$$

and after substituting (7) to (6)

$$
T=T_{0} e^{\frac{s}{c_{0}}}
$$

As can be noticed, a related general symbol has been introduced instead of a value of the initial temperature. It results from further intention where the energetic states of the solid will be discussed. The temperature $T_{0}$ is the initial temperature relating to the parameter of initial energetic state of the considered thermodynamic agent.

It appears the formulae (4) and (8) do not indicate the quantum nature of the phenomenon of the entropy growth in spite that it possesses such a nature. There is lack of determination of the second energetic state (the initial one has been marked). The second state refers to the moment when the body starts to enlarge its volume with no above mentioned condition referred to $(v=i d e m, d v=0)$. Therefore these state limits should be determined in view of noticing both quantum and energetic nature of the considered phenomenon. It would be absolutely impossible without a determination of these limits. This is why it was behind the cognition limits until now which has been reflected by some exemplary literature references [3-6].

\section{ADEQUATE DESCRIPTION OF THE CONSIDERED HEAT PHENOMENON}

At the source of this cognitive way of all physical phenomena, which occur with a variable rate/intensity or velocity between the neighbouring energetic states, there is a general differential description, namely:

$$
d Z= \pm \frac{\partial Z}{\partial N} d N
$$

where: $d Z$ - total differential of the magnitudes being the dependent variables, $d N$ - total differential of the magnitudes being the independent variables, $\partial Z / \partial N$ - partial derivative of the magnitudes dependent against independent ones. The signs $( \pm)$ are the algebraic operators fulfilling a determined function. The sign $(+)$ confirms the physical sense of a determined mathematic record, whereas the sign (-) assigns a physical meaning to the record.

That general source differential equation has been used in the past [7-9], where it is referred to the detailed reality, specifically of technological nature with different initial conditions. These conditions, the properly understood initial conditions determine the detailed 
solutions of that kind of equations. It concerns the initial conditions of the phenomenon or process, and not those connected with the beginning of measure of the determined physical magnitudes. The measurement of these magnitudes is necessary but not a sufficient condition. The sufficient condition refers to their measurements at the very beginning of the phenomenon.

The record of equation (9), applied to the considered phenomenon, possesses the following configuration:

$$
d T=\frac{\partial T}{\partial s} d s
$$

where, as may be noticed, the positive algebraic operator is maintained. That means the temperature changes are progressively rising and the speed/rate of these changes rises with the entropy growth.

The entropy-temperature characteristics of the process of the solid heating, with the accompanying phenomenon of the entropy growth of a solid, is surely interesting from the scientific viewpoint (Fig. 2). It possesses several cognitive features.

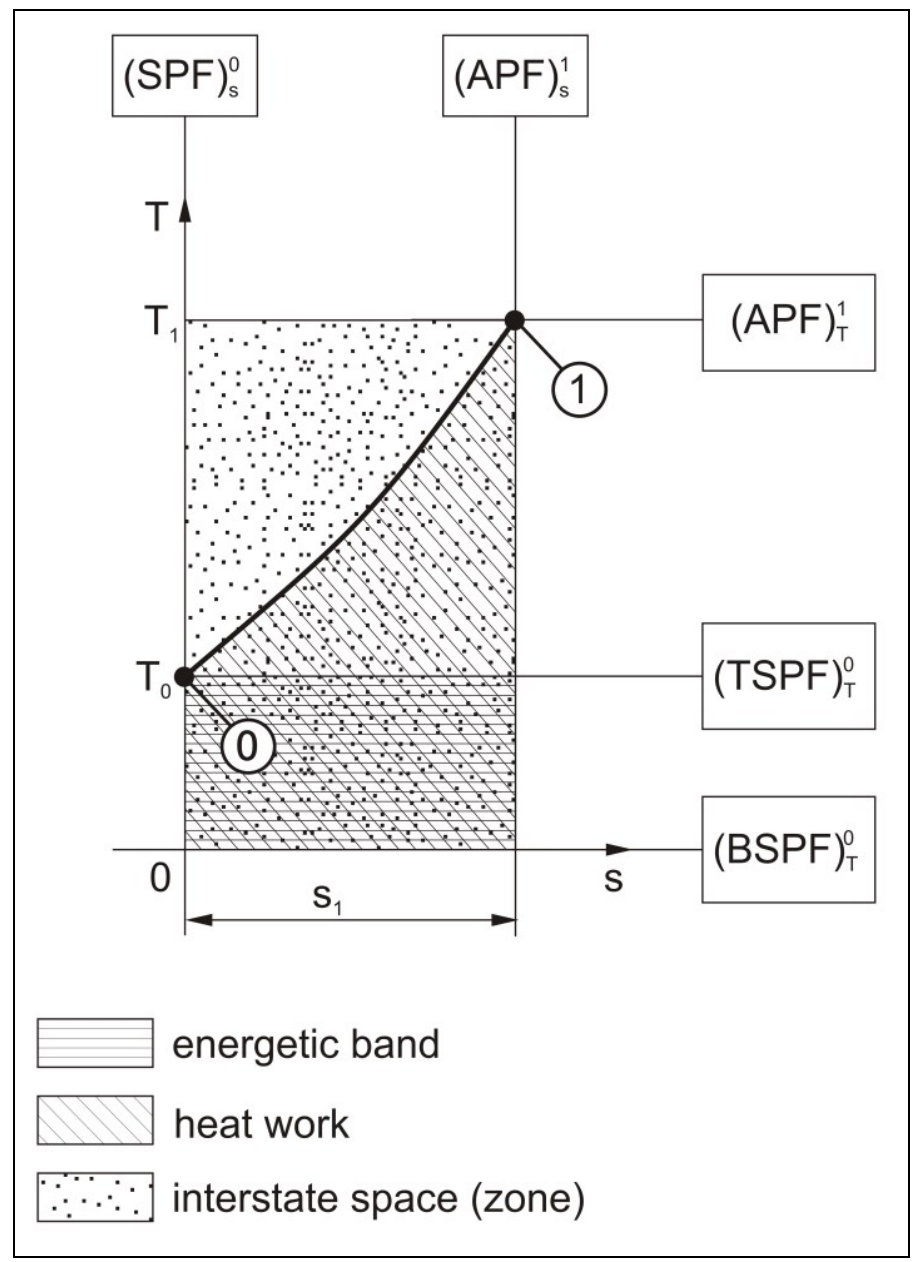

Fig. 2. Entropy-temperature characteristics of the process of solid heating 
In the assumed entropy-temperature coordinate system the dependence $T=f(s)$ reflects the progressively rising exponential curve. This curve (its course has an indicative character) is placed in the space/zone limited by the potential fields with the fields denoting energetic states of the heated body (dotted area in Fig. 2).

In the direction of entropy the limits (potential fields) of this area are as follows: entropy stable potential field $(S P F)_{s}^{0}$, entropy unstable potential field $(A P F)_{s}^{1}$. Additional symbols, the numerical ones, being in the powers, denote the reference of the field to a determined point, that is zero and the first one, respectively. There are more fields in the temperature direction and they are as follows: bottom temperature stable potential field $(B S P F)_{T}^{0}$, upper/top temperature stable potential field $(T S P F)_{T}^{0}$, temperature unstable potential field $(A P F)_{T}^{1}$. The digits $0 ; 1$, as mentioned above, denote ordering the fields to the determined points.

One may notice, that between the fields $(B S P F)_{T}^{0}$ and $(T S P F)_{T}^{0}$ there is the energetic band (the shaded area with horizontal lines in Fig. 2). That area refers to the own specific heat energy with the specific heat potential $e_{c}^{0}$ as the measure. That means

$$
e_{c}^{0}=T_{0} \cdot s_{1}
$$

where $s_{1}$ is the specific entropy related to the distance between the entropy potential fields, and $T_{0}$ being the initial temperature, that is $273.15 \mathrm{~K}$.

The operations on units indicate that the unit of the potential is $\mathrm{J}_{\mathrm{kg}}{ }^{-1}$. Therefore

$$
\left[e_{c}\right]_{S I}=K \frac{\mathrm{J}}{\mathrm{kg} \cdot K}=\mathrm{J} \cdot \mathrm{kg}^{-1}
$$

The proper/own heat energy of the considered thermodynamic agent, or of the solid, is its readiness to perform the heat work on it. That work corresponds with the oblique dashed area (see Fig. 2). The mentioned readiness is not a physical magnitude as yet, so the respective, explained above, the potential (energy measure) has been assigned to this intellectual magnitude (own heat energy). The specific heat work, as referred to the body mass, is bigger than the measure of the own heat energy. That is right because this work will be performed over the body.

The gained heat energy of a body is its ability to perform the heat work. The measure of the specific energy of this kind is the specific potential, determined as follows:

$$
e_{c}^{1}=T_{1} \cdot s_{1}
$$

where $T_{1}$ is the final temperature, related to the moment when the body increases its volume (below that temperature the body does not deform). 


\section{CREATION OF DETAILED CHARACTERISTICS OF THE ENTROPY GROWTH PHENOMENON}

The characteristics of phenomenon of the entropy growth is given by exponential, progressively rising curve, presenting the dependence of temperature $T$ on the specific entropy $s$ (Fig. 3).

$$
\mathrm{T}=\mathrm{T}_{0}+\left(\mathrm{T}_{1}-\mathrm{T}_{0}\right)\left(\mathrm{e}^{\frac{\mathrm{s}}{\mathrm{s}_{0}}}-1\right)
$$

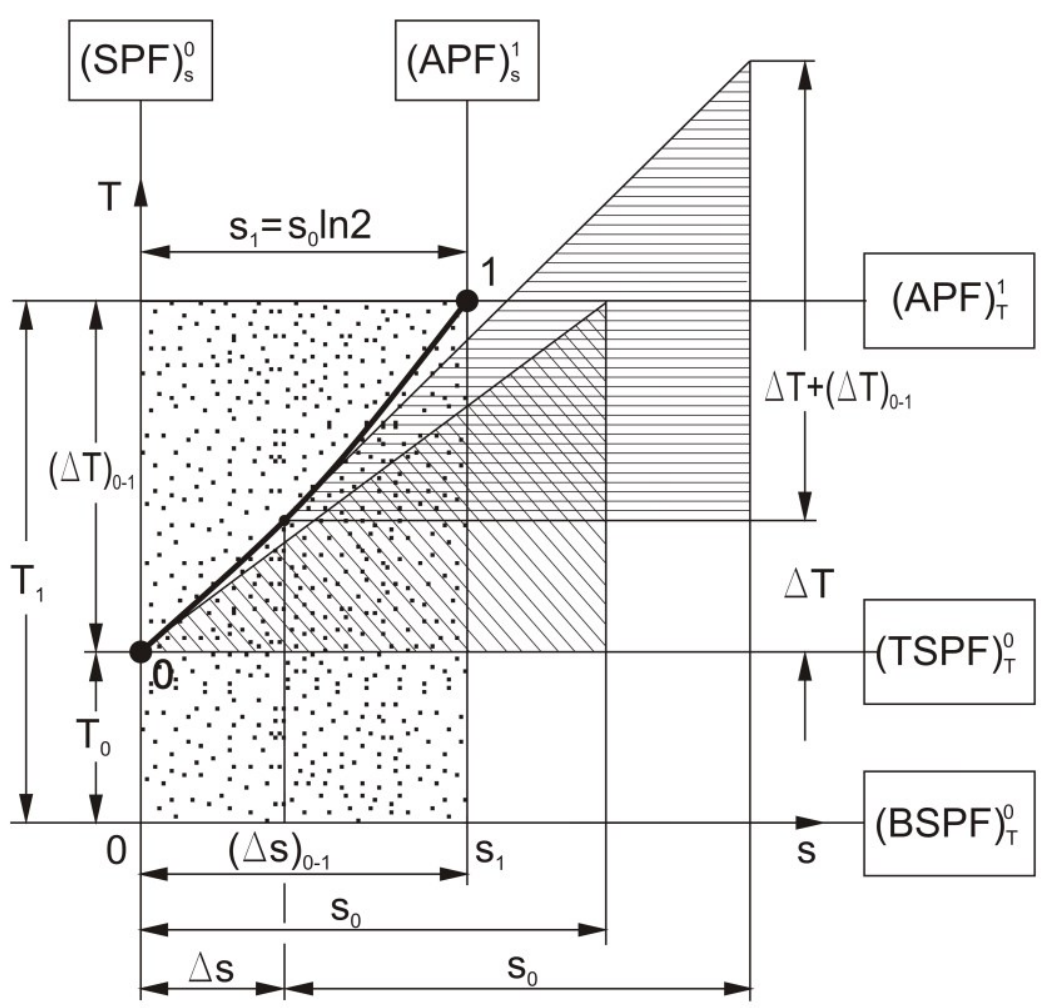

Fig. 3. Illustration of entropy-temperature characteristics creation schematic

This curve is the envelope of right-angled triangles, with the entropy shorter side/leg being constant and equaled to the so-called entropy constant $s_{0}$, whereas the temperature leg changes respectively. The initial point of the curve is on the crossing of the stable potential fields: entropy stable potential field $(S P F)_{s}^{0}$ and the top temperature stable potential field $(T S P F)_{T}^{0}$. The final point of this curve is situated on the crossing of unstable potential fields: the first entropy unstable potential field $(A P F)_{S}^{1}$ and the first temperature unstable potential fields $(A P F)_{T}^{1}$. The limits of the entropy-temperature space are the mentioned entropy fields, 
the mentioned unstable temperature potential field $\left(A P F_{T}^{1}\right)$ and the bottom temperature stable potential field $(B S P F)_{T}^{0}$, which the field is placed on the zero temperature level (see Fig. 3 ).

The temperature leg of this moving right-angled triangle is, as mentioned above, respectively variable that results from the changing position of tangent to the temperature curve. For $\Delta T$ the length of that leg equals $\Delta T+(\Delta T)_{0-1}$.

Now one may start with integrating the equation (10), denoting the limits of integrals from the total differentials. That means

$$
\int_{T_{0}+\Delta T}^{T_{0}+2 \Delta T+(\Delta T)_{0-1}} d T=\frac{\partial T}{\partial s} \int_{\Delta s}^{\Delta s+s_{0}} d s
$$

and then

$$
\Delta T+(\Delta T)_{0-1}=\frac{d T}{d s} s_{0}
$$

or

$$
\frac{d T}{\Delta T+(\Delta T)_{0-1}}=\frac{1}{s_{0}} d s
$$

It may be noticed that the partial derivative has been substituted by the quotient of the total differentials (diminutives). It could be done that way because the total differentials have been clearly determined by introducing limits of the integrals.

Furthermore, by integrating both sides of the equation (15), one obtains the result

$$
\ln \left[\Delta T+(\Delta T)_{0-1}\right]=\frac{1}{s_{0}} s+C^{*}
$$

that is

$$
\Delta T+(\Delta T)_{0-1}=e^{\frac{s}{s_{0}}+C^{*}}=e^{C^{*}} \cdot e^{\frac{s}{s_{0}}}=C e^{\frac{s}{s_{0}}}
$$

After regarding that for $s=0$ the magnitude $\Delta T=0$, one obtains

$$
C=(\Delta T)_{0-1}
$$

and after substituting (18) to (17)

$$
\Delta T=(\Delta T)_{0-1}\left(e^{\frac{s}{s_{0}}}-1\right)
$$


that is (see Fig. 3)

$$
T=T_{0}+\Delta T=T_{0}+(\Delta T)_{0-1}\left(e^{\frac{s}{s_{0}}}-1\right)
$$

or

$$
T=T_{0}+\left(T_{1}-T_{0}\right)\left(e^{\frac{s}{s_{0}}}-1\right)
$$

One may determine now the second coordinate of the point 1, which is the entropy related to the temperature, from which the deformation of the considered thermodynamic agent begins, and with this the specific volume as well. That result has been obtained by introducing the temperature $T=T_{1}$ and the specific entropy $s=s_{1}$ to equation (21). Thus

$$
s_{1}=s_{0} \ln 2
$$

Now there is a consecutive functional characteristics, being the formula on the heat work

$$
L_{c}=\int_{0}^{s_{1}} T(s) d s
$$

That work (and not the energy, nor its measure, that is the potential) is expressed by the relationship

$$
L_{c}=\int\left[T_{0}+\left(T_{1}-T_{0}\right)\left(e^{\frac{s}{s_{0}}}-1\right)\right] d s=s_{1}\left(2 T_{0}-T_{1}\right)+s_{0}\left(T_{1}-T_{0}\right) e^{\frac{s}{s_{0}}}
$$

One can see, there is an immense difference between the heat work and the measure of the heat energy, which is the heat potential. They are not the magnitudes which could be identified. Moreover, it results from these considerations that the energy notion (here - the heat energy) really exists and has been defined clearly and distinctly.

It evidently results from these analyses and description that the considered phenomenon has the quantum character with the changes occurring between the neighbouring energetic states, determined by the coordinates $\left(s=0, T=T_{0}\right)$ and $\left(s=s_{1}, T=T_{1}\right)$. That heat potential is the quantum (portion) of the heat energy; both the own one $e_{c}^{0}$, and then the gained one $e_{c}^{1}$.

Now one may determine the dependence between the specific heat $c_{0}$ and the entropy constant $s_{0}$. It is possible, thanks to the scheme (Fig. 4), to derive that dependence. The entropy constant is positioned on the upper/top temperature stable potential field $(T S P F)_{T}^{0}$, 
whereas the specific heat is positioned on the bottom temperature stable potential field $(B S P F)_{T}^{0}$.

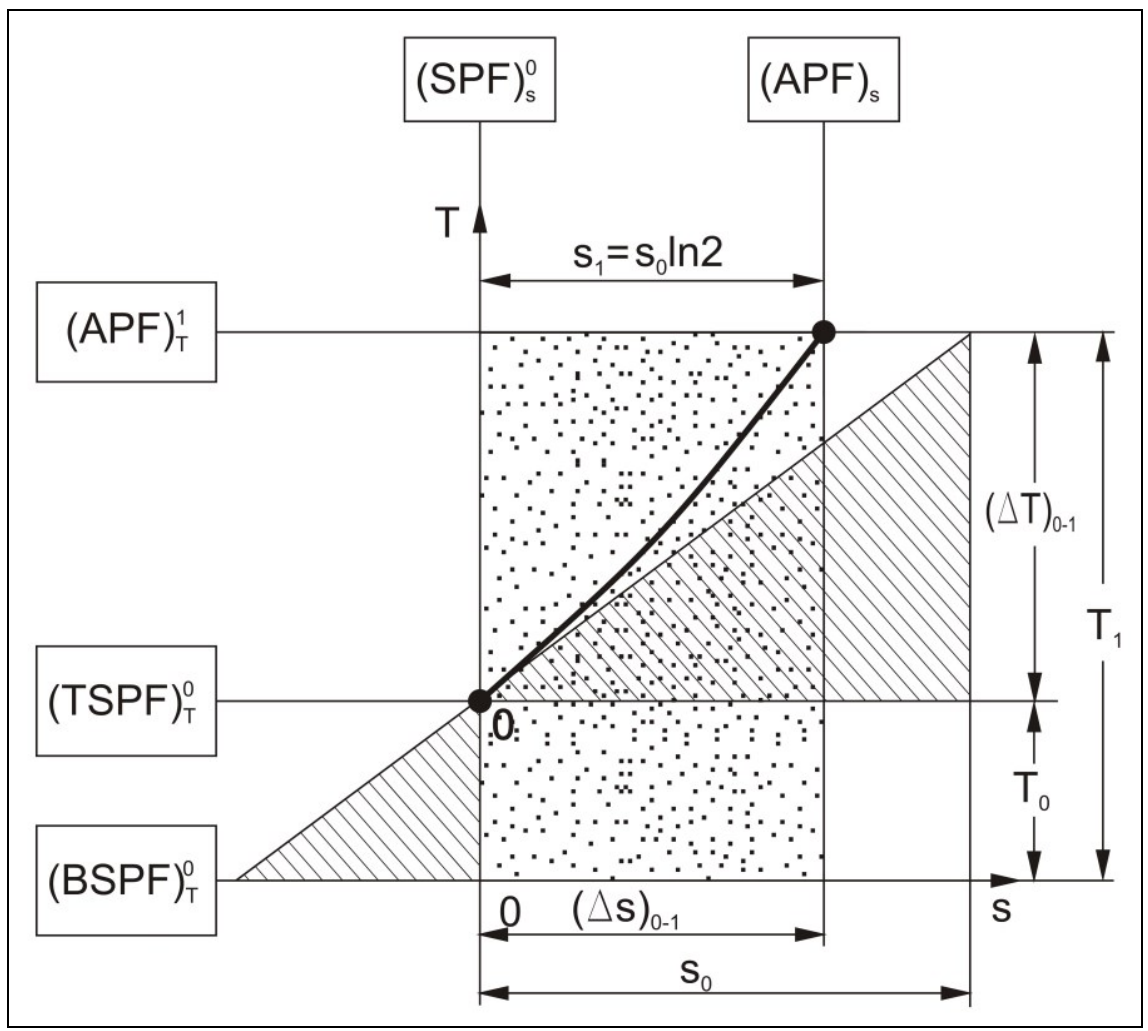

Fig. 4. Scheme of derivation of dependence between the specific heat $c_{0}$ and the entropy constant $s_{0}$

The tangent to the curve $T=f(s)$ in its zero point creates two right-angled triangles (oblique dashed) on the net of all potential fields which, as can be seen, are the similar triangles. Thus they are characterized by the following proportion:

$$
\frac{s_{0}}{c_{0}}=\frac{T_{1}-T_{0}}{T_{0}}
$$

from which it results that

$$
s_{0}=c_{0} \frac{T_{1}-T_{0}}{T_{0}}
$$

Now (knowing the values $c_{0}, T_{0}, T_{1}$ ) one may determine the entropy constant $s_{0}$, that will allow to express, for a determined material, the dependence $T=f(s)$. 


\section{CONCLUSION}

In conclusion, it is worth noting that the essential feature of the considered phenomenon has been discovered. It is about the quantum nature and it appears that the reality, as considered in the macroscopic scale, also possesses quantum energetic nature. Thus not only the microscopic world is quantified with the extended quantum theory.

It is not possible to notice the quantum nature of a determined phenomenon if no energetic states are perceived which determine the quantum behaviour of the body (thermodynamic agent). In reference to a solid this non-determined (or ignored) state limit is determined by the temperature $T_{1}$, corresponding with the moment of the occurrence of the specific volume increment. That temperature may be read out of the dilatometric curve (accurately done), on which the segment of temperatures referred to the situation where there is no incremental changes of the body geometry, is visible. (One may add that this peculiar inertia refers not only to the thermal phenomena; it refers also to the mechanical reality.)

It results from the presented considerations that the energy notion exists together with its definition. It is worth adding that this notion is comprehensible and may function on the grounds of science, not only as an abstraction. Let us add it to the known notion of entropy, which the notion is so exactly explained in the exemplary literature [1-2, 10-12].

The work has been concerned on the determined thermal sphere of the reality, but it may be the incentive to discover and develop the quantum nature also in other realities. The existent knowledge leaves much to improve the energetic aspects of description of microscopic reality. It is worth filling up this gap by undertaking the activity to change the state.

\section{NOMENCLATURE}

$c_{0}-$ specific heat

$s_{0}$ - entropy constant

$v$ - specific volume

$T$ - temperature

$T_{0}$ - initial temperature

$T_{1}$ - final temperature

$d s$ - total differential of entropy

$d T$ - total differential of temperature

$\partial T / \partial s-$ partial derivative of temperature against entropy

$e_{c}^{0}-$ proper specific heat potential

$e_{c}^{1}-$ gained specific heat potential

$(S P F)_{s}^{0}$ - entropy stable potential field

$(A P F)_{s}^{1}$ - entropy unstable potential field

$(B S P F)_{T}^{0}$ - bottom stable temperature potential field

$(T S P F)_{T}^{0}$ - top stable temperature potential field

$(A P F)_{T}^{1}$ - temperature unstable potential field 


\section{References}

[1] P. Attkins, Galileo finger (in Polish). Publ. House REBIS, Poznań 2006 (transl. from English), 1st edition.

[2] S. Ochęduszko, Applied thermodynamics (in Polish). WNT, Warszawa 1967, 2nd edition, amended and supplemented.

[3] P. M. Harman, Energy, force, and matter: The conceptual development of nineteenthcentury physics, Cambridge University Press, 1982.

[4] B. Lightman (ed), Victorian science in context, University of Chicago Press, 1997.

[5] C. Smith, The science of energy: A cultural history of energy physics in Victorian Britain, Athlone Press, London 1998.

[6] J. Barbour, The discovery of dynamics, Oxford University Press, 2001.

[7] Zdzisław Pluta, Fundamentals of surface smoothing with elastic wheels (in Polish). Ed by Politechnika Koszalińska, Koszalin 2007.

[8] Zdzisław Pluta, Tadeusz Hryniewicz, Intern. J. Adv. Manuf. Technol. 62 (2012) 529-542.

[9] Zdzisław Pluta, Tadeusz Hryniewicz, J. Modern Physics 3(8) (2012) 793-802.

[10] P. Atkins, The second law, W. H. Freeman, New York 1987.

[11] P. Coveney, R. Highfield, Time arrow/loop: how to develop the biggest mystery of science. Zysk i S-ka, Poznań 1997 (transl. from English).

[12] P. Atkins, J. de Paula, Physical chemistry, Oxford University Press and W. H. Freeman, New York 2002. 